\title{
Apical MUC1 expression revealed on the foveolar epithelium in $H$. pylori gastritis
}

\author{
M Rashid ${ }^{1}$, A S Teixeira ${ }^{2}$, U Qureshi ${ }^{1}$, S P Pereira ${ }^{3}$, M R Novelli ${ }^{4}$ and D M Swallow ${ }^{\star 2}$ \\ ${ }^{1}$ Department of Oncology, UCL Cancer Institute, University College London, 72 Huntley Street, London WCIE 6BT, UK; ${ }^{2}$ Research \\ Department of Genetics, Evolution and Environment, University College London, Darwin Building, Gower Street, Gower Street, \\ London WC1E 6BT, UK; ${ }^{3}$ UCL Institute for Liver and Digestive Health, University College London, Royal Free Hospital Campus, \\ Rowland Hill Street, London NW3 2QG, UK and ${ }^{4}$ Department of Histopathology, University College Hospital London NHS \\ Foundation Trust, Rockefeller Building, University Street, London WCIE 6JJ, UK
}

Background: The membrane mucin MUC1 is altered in its pattern of expression in cancer, and also in other pathological situations, including Helicobacter pylori gastritis. Here we investigate the basis for the loss of apical staining of the gastric foveolar epithelium in $\mathrm{H}$. pylori gastritis.

Methods: MUC1 was examined in the gastric antrum from cases of $H$. pylori gastritis and normal controls. We used tissue sections that were either treated or not treated with periodate to effect deglycosylation, and the monoclonal antibodies LICRLonM8, MUSE-11, CT2 and BC2.

Results: We show that the epitopes on the TR domain of MUC1 are partially cryptic due to glycosylation and that MUC1 is present on the apical surface of the gastric foveolar epithelium of gastritis patients.

Conclusion: This observation suggests that there is no substantial loss of the mucin domain of MUC1 from the apical surface in gastritis, as suggested by others, but rather the H. pylori influences the glycosylation of MUC1. This paper highlights the issue of epitope specificity of monoclonal antibodies directed against disease-associated markers, specifically when they are glycoproteins, as is the case for many cancer markers.

It has frequently been reported that the membrane mucin MUC1 is altered in its pattern of expression in cancer, and MUC1 is thus a well-known 'cancer marker' (Taylor-Papadimitriou et al, 1999; Kufe, 2009). Changes in detectable MUC1 have multiple causes: altered glycosylation and also altered subcellular location being as important as altered gene expression. MUC1 has been widely used as a target in many diagnostic and therapeutic developments, some of which have been useful while others have been of variable benefit (Kufe, 2009; Beatson et al, 2010; Mukhopadhyay et al, 2011; Rong et al, 2012). These variable outcomes undoubtedly are partly because changes in MUC1 expression have sometimes been mis-interpreted. One of the problems has been that most antibodies to MUC1 detect peptide epitopes on the central tandem repeat domain and their binding is influenced by the glycosylation of the TR domain.
MUC1 expression is also altered in other pathological situations. Our group has previously shown that the pattern of expression of MUC1 on the gastric foveolar epithelium is altered in Helicobacter pylori gastritis, a strong risk factor for gastric cancer. Using two different antibodies directed against the peptide backbone of the TR domain of MUC1, we showed that there was loss of apical staining for the extracellular domain and increase in intracellular staining (Vinall et al, 2002), while the cytoplasmic domain could be detected on the apical surface using a polyclonal antiserum directed against the cytoplasmic tail. The absence of apical staining in gastritis may be due to several reasons: (i) alteration of the glycosylation of the mucin domain may make the tandem repeat epitope cryptic; (ii) the mucin domain may somehow be internalised into the cell, leaving the cytoplasmic domain on the 
surface; or (iii) the MUC1 ectodomain may be lost from the surface through cleavage by $H$. pylori.

It has more recently been shown that $H$. pylori adhere to purified MUC1 (Linden et al, 2004b), and that mice lacking MUC1 are colonised five-fold more in one day of infection than mice with MUC1, and that they develop atrophic gastritis (McGuckin et al, 2007). It was thus hypothesised that MUC1 acts as a decoy limiting binding of the $H$. pylori to the cell surface, and that this leads to the shedding of the extracellular domain of MUC1 that is loaded onto the $H$ pylori (McGuckin et al, 2007). Studies using a gastric cell line report that shedding can indeed be induced by beads used as the decoy, and that MUC1 detection is reduced on infection and can be found bound to the H. pylori (Linden, 2009). However, reduction in apical MUC1 staining in $H$ pylori gastritis might alternatively (or also) reflect the cryptic nature of the epitope detected, due to glycosylation.

Here we aim to provide an insight into the interpretation of changes in MUC1 expression in this cancer-predisposing condition, and explore the possibility that the differences in detection reflect changes in glycosylation.

\section{MATERIALS AND METHODS}

Biopsy specimens were taken endoscopically from the gastric antrum of patients at University College London Hospitals. All patients gave fully informed consent, and the study was approved by the local ethical committee (UCL/UCLH 01/0237). The preliminary studies were done using samples collected previously (Vinall et al, 2002) originally under ethics UCL/UCLH 95/3037. The biopsy samples were processed using routine UCLH procedures - immediate fixation in standard formal saline at ambient temperature and same-day transfer to the laboratory for machine-automated processing, starting with formal saline and passing through standard alcohols and xylene to paraffin wax embedding, using the machine timings for biopsies. Sections, $3 \mu \mathrm{m}$, were mounted on SuperFrost Plus microscope slides and deparaffinised in Histoclear followed by rehydration with graded alcohols and water. Gastritis was classified histologically using the Sydney System (Dixon et al, 1996), and H. pylori status was determined using the CLO (Campylobacter-like organism) test. Histology was examined under $\mathrm{H} \& \mathrm{E}$ and immunostaining as described previously (Vinall et al, 2002). Biopsy specimens from two groups of patients were tested based on these results: group 1, histologically normal samples with no $H$. pylori infection; group 2, samples from individuals with current gastritis who were H. pylori-positive.

We used three monoclonal antibodies (LICRLonM8, BC2 and MUSE-11) directed against different epitopes on the tandemly repeated 20-amino-acid sequence, VTSAPDTRPAPGSTAPPAHG, of the peptide backbone MUC1. LICRLonM8 (McIlhinney et al, 1985) recognises the epitope DTR (Price et al, 1990), whereas BC2 recognises RPAP (Xing et al, 1992), but both of these reagents can apparently recognise fully glycosylated MUC1 (Price et al, 1985; Xing et al, 1989). MUSE-11 has been shown to bind best to the amino-acid motif PDTRPAPG (Hinoda et al, 1990, 1993), but on immunohistology is cryptic in gastric samples from most individuals without prior deglycosylation (Bara et al, 1993). MUSE-11 was selected as it provides a control for the deglycosylation procedure. It should however be noted that MUSE-11 does show staining without special treatment, in most non-secretor individuals - people who are homozygous for the deficiency of the $\alpha-2$ fucosyltransferase, FUT2 and do not carry the Lewis $b$ antigen $(\operatorname{Le} a+b-)$. We also used an Armenian hamster monoclonal antibody, CT2, directed against the last 17 amino acids of MUC1, SSLSYTNPAVAATSANL, which is uniquely found in the intracellular cytoplasmic tail of MUC1 (Schroeder et al, 2001; Croce et al, 2003).

Each of the antibodies was titrated, the effect of heat-induced epitope retrieval with citrate buffer $\mathrm{pH} 6.0$ tested, and conditions chosen, to obtain maximum specificity and sensitivity. The final conditions used were for LICRLonM8, 1 in 1000 culture supernatant, MUSE-11 $\left(0.4 \mu \mathrm{g} \mathrm{ml}^{-1}\right)$ and BC2 $\left(1.4 \mu \mathrm{g} \mathrm{ml}^{-1}\right)$, and for CT2, 1 in 250 culture supernatant, and no epitope retrieval. Endogenous peroxidase activity was blocked by incubating sections in $3 \%$ hydrogen peroxide made up in methanol. For LICRLonM8 and MUSE-11, sections were incubated in 5\% normal goat serum for $20 \mathrm{~min}$ to reduce nonspecific binding, followed by overnight incubation at $4{ }^{\circ} \mathrm{C}$, with primary antibody diluted in $5 \%$ normal goat serum. Bound antibody was detected using the Dako REAL Envision (Dako, Glostrup, Denmark) detection system and the reaction was visualised using the Dako REAL DAB + chromogen. Sections were washed in distilled water, counterstained with haematoxylin, dehydrated through graded alcohols and cleared through Histoclear, before mounting with DPX-mountant. For CT2 and BC2 detection, sections were incubated with either the CT2 or the BC2 antibody for $60 \mathrm{~min}$ followed by a $30-\mathrm{min}$ incubation with $7.5 \mu \mathrm{g} \mathrm{ml}^{-1}$ biotinylated goat-anti-Armenian Hamster (Jackson Laboratories, West Grove, PA, USA) or 1 in 200 biotinylated horse-anti-mouse (Vector Laboratories) secondary antibody, respectively, and then a 40-min incubation with Vectastain ABC reagent (Vector Laboratories, Peterborough, UK) followed by colour development with $\mathrm{DAB}+$ reagent (Dako). For CT2, non-specific binding was blocked with $5 \%$ normal goat serum (Dako) and for BC2, non-specific binding was blocked with $3 \%$ normal horse serum.

To examine the effect of deglycosylation, antibodies were tested without or with periodate treatment before performing immunohistochemistry. Tissue sections were incubated in $20 \mathrm{~mm}$ periodic acid prepared in $0.05 \mathrm{M}$ acetate buffer, $\mathrm{pH} 5.0$, for $45 \mathrm{~min}$. After three 3-min washes in PBS/Tween 20 buffer (wash buffer), sections were incubated in $1 \%$ glycine for $30 \mathrm{~min}$ to neutralise acidic groups before being washed again in the same wash buffer. Immunohistochemistry was then performed. Additional controls for periodate treatment consisted of sections treated in the same way but omitting the primary antibodies. All incubations, unless otherwise stated, were done at room temperature. Treatment and staining was performed in five batches, in which the investigators were blinded to diagnosis, but all batches were found to contain both gastritis cases and controls. The pattern and intensity of staining were scored independently in all of the samples by two investigators (MR and DMS). The variation in intensity of staining was classified as weak (1), moderate (2) or strong (3), on the apical surface and intracellularly. The area of staining was semiquantitatively assessed by inspecting all possible fields under a $\times 20$ objective and taking an average. This was done separately for the foveolar epithelium of the gastric pits and the region extending from the isthmus to the glands. Mean scores, of the two observers, for each measurement were used for statistical analyses. Results were analysed in SPSS 20, using rank tests as the data were non-normally distributed.

Standard serological tests with anti-Le ${ }^{\mathrm{a}}$ and $\mathrm{Le}^{\mathrm{b}}$ were previously done using blood samples from our earlier cohort of individuals. Secretor status was deduced from the Lewis phenotype in Lewispositive patients. This information was not available for the current cohort.

\section{RESULTS}

In preliminary studies, we examined some of the same tissue samples as used in our previous study, using the monoclonal 
antibodies LICRLonM8 and two other antibodies directed against the tandem repeat domain, BC2 and MUSE-11, and also CT2 to the cytoplasmic tail. As before, LICRLonM8 showed strong apical staining of the epithelial cells in the normal controls, while no apical staining was seen in the $H$. pylori gastritis samples, even though there were areas of strong cytoplasmic staining (Figures 1A and C). Similar results were obtained with BC2 (data not shown). In contrast, apical staining was seen with $\mathrm{CT} 2$ in six $H$. pylori gastritis cases as well as in three normal controls, and the results obtained confirmed those obtained previously with the polyclonal serum, to the cytoplasmic tail (Figures $1 \mathrm{~B}$ and D).

With MUSE-11, which is known to recognise an epitope that is masked by glycosylation in most individuals, and appears to depend on blood group and secretor status (Bara et al, 1993), we initially tested 15 samples from the previous cohort in which secretor status was known. None of the eight secretors we tested (including two with active $H$. pylori gastritis) showed staining (Figure 1E). Six out of seven non-secretors (including two with active $H$. pylori gastritis) showed clear apical staining of the foveolar epithelium (Figure 1G). Chemical deglycosylation showed the reappearance of the MUSE-11 epitope in the secretors, as previously reported by others (Bara et al, 1993), confirming the efficacy of the deglycosylation procedure (Figure $1 \mathrm{~F}$ ).
A new cohort of 20 cases of $H$. pylori gastritis cases and 14 biopsies assessed as normal were tested using the two monoclonal antibodies LICRLonM8 and MUSE-11 using prior deglycosylation treatment and adjacent sections with no such treatment.

Apical LICRLonM8 staining was found on the foveolar epithelium in all normal but not in most $H$. pylori gastritis cases, agreeing with previous observations (Figures $1 \mathrm{I}$ and $\mathrm{K}$ and $2 \mathrm{~A}$ ). The staining in the normal cases extended over a variable proportion (median 60\%) of the gastric pit, but appeared more extensive and intense on the surface (see Figure1I). When quantified in the same way, the distribution in the gastritis cases gave a median of $0.1 \%$ (Figure $1 \mathrm{~K}$ ). This difference between cases and controls was highly statistically significant $(P<0.0001)$ and there was a corresponding difference in intensity (Figures 1I and $\mathrm{K}$ ). Cytoplasmic (perinuclear) staining was also seen, in contrast, being prominent, though somewhat patchy, in both controls (median 43\%) and in the H.pylori gastritis cases, which gave a slightly higher median distribution of $46.5 \%$. This difference between cases and controls was, however, not statistically significant $(P=0.478$, Figure $2 \mathrm{~B})$, nor was there any significant difference in intensity. The distribution of apical staining in the isthmus and glandular region was not as widespread as in the
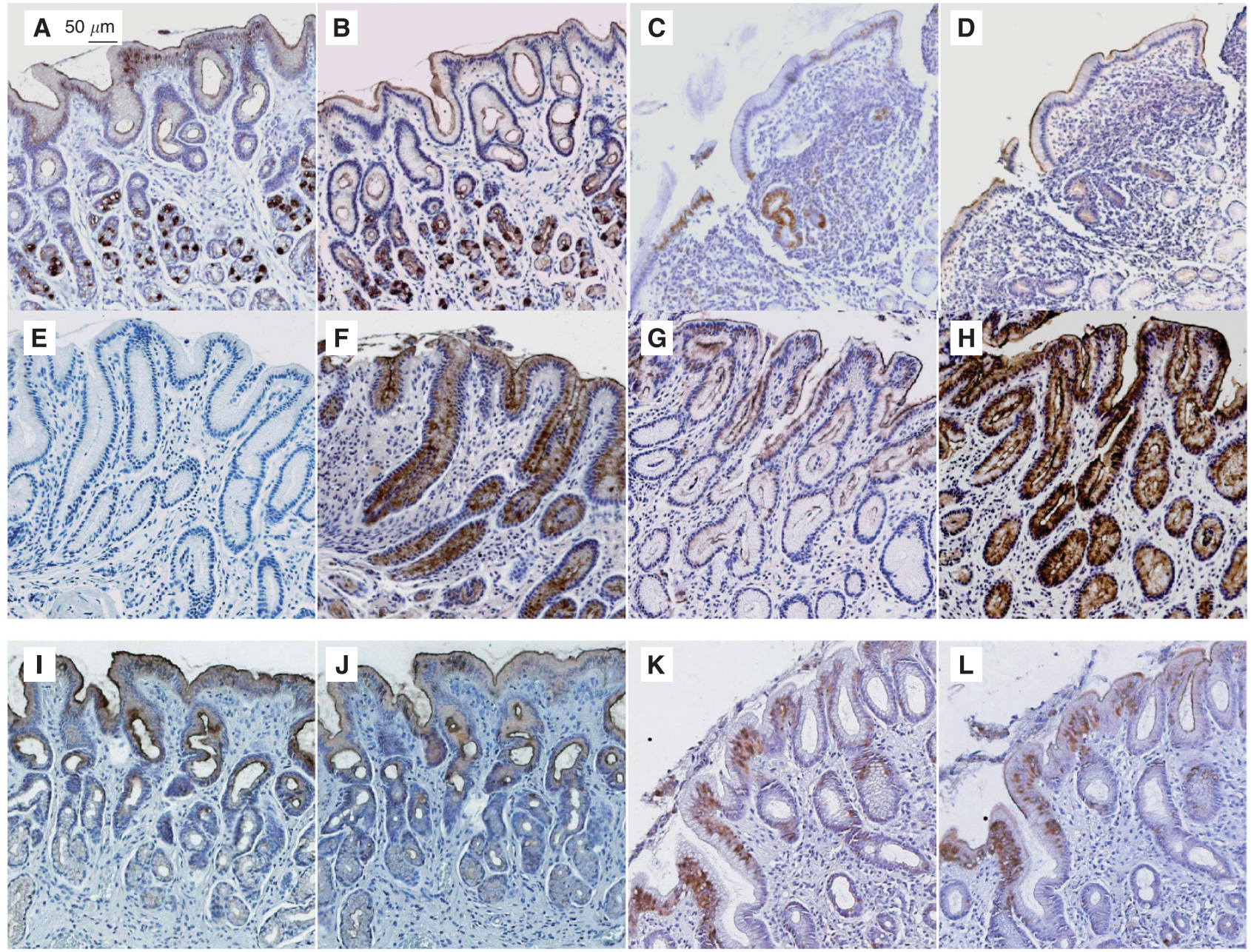

Figure 1. Detection of MUC1 in antral epithelium from normal and gastritis cases using antibodies against different domains. Sections $\mathbf{A}$ and $\mathbf{C}$ are stained with LICRLonM8, while for $\mathbf{B}$ and $\mathbf{D}$ the antibody CT2 against the cytoplasmic domain is used. Sections $\mathbf{A}$ and $\mathbf{B}$ are from a normal biopsy while $\mathbf{C}$ and $\mathbf{D}$ are from an $\mathbf{H}$.pylori gastritis case. Sections $\mathbf{E}$ to $\mathbf{H}$ are stained with MUSE-11. Sections $\mathbf{E}$ and $\mathbf{F}$ are from a secretor individual, and $\mathbf{G}$ and $\mathbf{H}$ from a non-secretor. Sections $\mathbf{E}$ and $\mathbf{G}$ are not deglycosylated by periodate treatment, while $\mathbf{F}$ and $\mathbf{H}$ are deglycosylated. Sections I to $\mathbf{L}$ are stained withLICRLonM8. Sections $\mathbf{I}$ and $\mathbf{J}$ are from a normal, while $\mathbf{K}$ and $\mathbf{L}$ are from an $\mathbf{H}$. pylori gastritis case. Sections $\mathbf{I}$ and $\mathbf{K}$ are not deglycosylated while $\mathbf{J}$ and $\mathbf{L}$ are deglycosylated. Setion $\mathbf{A}$ shows the scale bar corresponding to the magnification of all sections. 

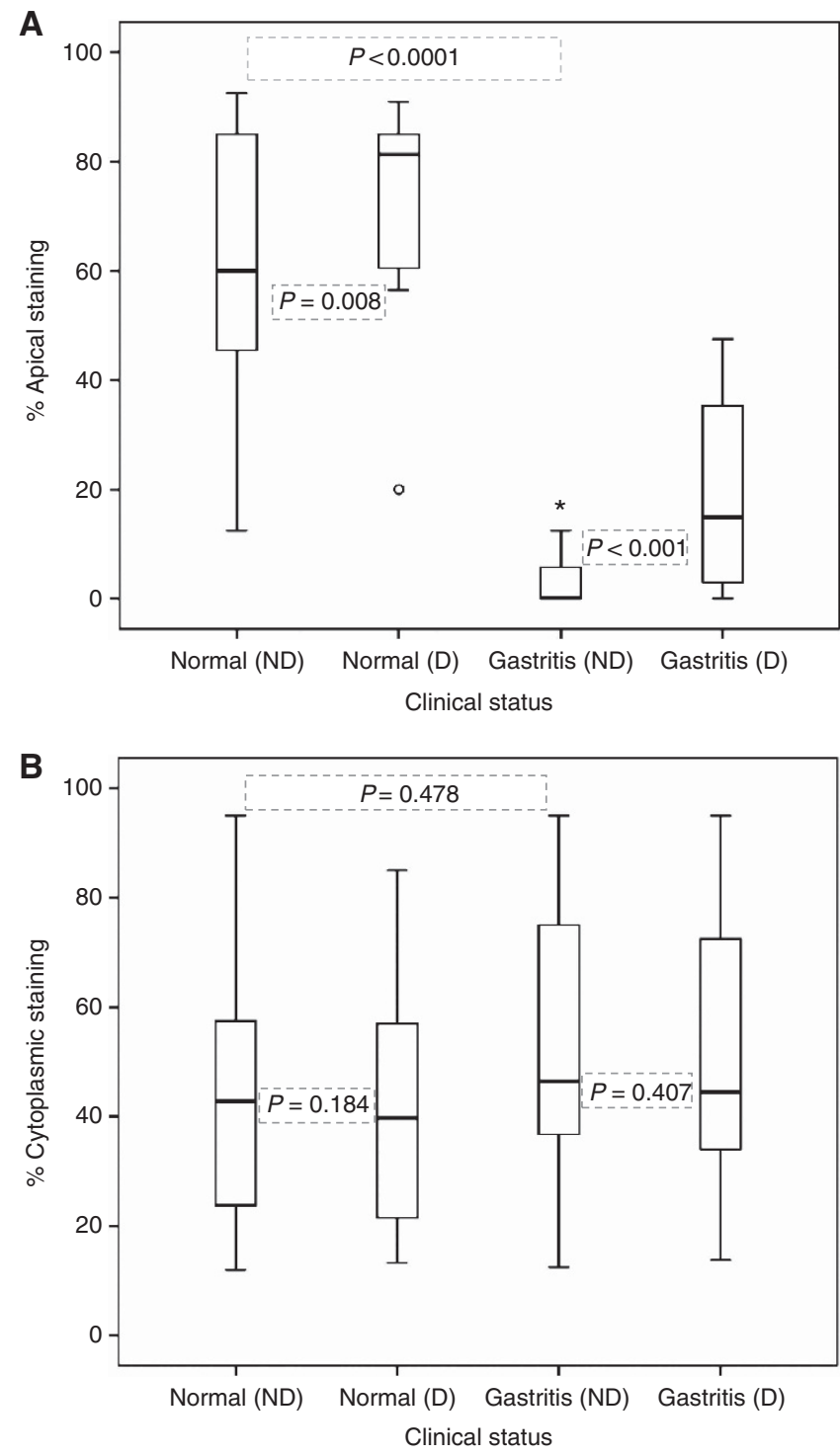

Figure 2. Relative distribution of LICRLonM8 apical staining within the gastric pits of normal and gastritis cases and the effect of periodate treatment. (A) Apical staining. (B) Cytoplasmic staining. The Mann-Whitney $U$ test was used to compare the cases and controls, while the related-samples Wilcoxon signed rank test was used to assess the effect of treatment. P-values are shown in the figure.

$\mathrm{D}=$ deglycosylated; $\mathrm{ND}=$ non-deglycosylated.

gastric pit, but again there was significantly less staining in $H$. pylori gastritis cases than in normal controls $(P<0.0001)$ (data not shown) with no significant difference of the cytoplasmic staining.

Deglycosylation treatment caused re-appearance of apical staining with LICRLonM8 in most of the gastritis cases (Figures $1 \mathrm{~L}$ and $2 \mathrm{~A}$ ), but there was also a significant increase in some of the controls (Figure 2A). The cytoplasmic staining was in contrast slightly reduced, but this was not statistically significant (Figure 2B).

MUSE-11 showed little or no staining of the foveolar epithelium in the majority of $H$. pylori gastritis cases or controls (as in Figure $1 \mathrm{E})$, with clear extensive apical staining in just a few $(n=2$ for $H$. pylori gastritis and $n=6$ for normals). It seems likely that these few individuals were non-secretors. After deglycosylation, apical staining of the foveolar epithelium was seen in all normal

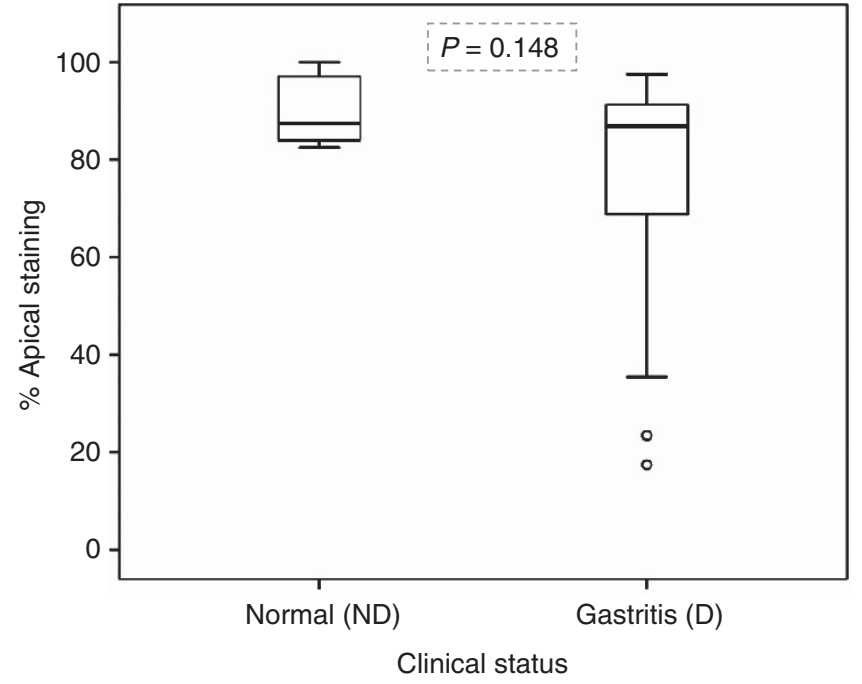

Figure 3. Relative distribution of MUSE-11 apical staining in the gastric pits of normal and gastritis cases after periodate pretreatment (D). The difference in apical immunostaining between normal and gastritis cases was not statistically significant $(P=0.148)$. Statistical analysis was performed by using the independent-samples Mann-Whitney $U$ test.

controls (median distribution $89 \%$, mean intensity 2.5 ), and also in all H. pylori gastritis cases (median distribution 88\%, mean intensity 1.9), but the difference between cases and controls was not statistically significant $(P=0.148$, Figure 3$)$.

BC2 was tested on six cases and six controls and also showed very scant apical staining of the foveolar epithelium in 5 out of 6 of the gastritis cases, which was in all cases greatly increased by deglycosylation (Supplementary Figure 1) compared with extensive staining in the normal controls without the need for deglycosylation.

\section{DISCUSSION}

The distinct difference observed previously (using LICRLonM8) between the pattern of expression of MUC1 on the gastric epithelium of normal controls and that of patients with $H$. pylori gastritis was replicated in this study. By making use of periodate treatment, we show that MUSE-11, a MUC1 antibody that is known to detect an epitope that is totally cryptic to glycosylation in most individuals, gives a very strong apical signal in all $H$. pylori gastritis patients as well as in all normal controls. The increase in apical staining observed with LICRLonM8 after periodate treatment also suggests that this epitope is at least partially cryptic, particularly in H. pylori gastritis, and it is noteworthy that the same effect was seen with BC2. Thus, by deglycosylation of the tissue sections we show that the TR domain of MUC1 is certainly present on the apical surface of gastric epithelial cells in $H$. pylori gastritis as well as in controls, showing that there is a gastritis-related change in a glycoprotein epitope.

Although there was no statistically significant difference in the distribution and intensities of intracellular staining between cases and controls, the data do show a trend of greater detection in the $H$. pylori gastritis cases compared with normal controls, consistent with our previous results (Vinall et al, 2002). In this case deglycosylation does not increase the signal detected, perhaps not surprisingly, because the MUC1 in the perinuclear region is likely to be less glycosylated, and therefore more accessible to the LICRLonM8 antibody. 
The combined observations suggest that $H$. pylori infection alters the peripheral glycosylation of the MUC1, which leads to much reduced detection by LICRLonM8 on the apical surface. It remains possible, however, that as suggested by Linden et al (2009)), H. pylori may also promote some shedding of the extracellular domain into the lumen of the stomach, as we detected slightly less MUC1 even after deglycosylation.

It is interesting to speculate how the changes in glycosylation are induced by $H$. pylori, and what kind of change could cause the epitopes to become more cryptic than in the uninfected epithelium. It suggests the non-intuitive scenario of increased glycosylation of certain side chains during infection. This is in fact consistent with the observations of Ota et al (1998), who showed (but do not comment on this) less Tn (GalNAc-attached direct to Ser/Thr) on the apical surface of antral foveolar epithelium during infection, but restoration after antibiotic treatment, implying that this core epitope had become cryptic during infection.

It is relevant to note that $H$. pylori BabA adhesin binds to the fucosylated $\mathrm{Le}^{\mathrm{b}}$ and $\mathrm{H}$ type 1 structures, which are located on MUC1 (Linden et al, 2004a, b), and MUSE-11 epitope is cryptic in $\mathrm{Le}^{\mathrm{b}}$-carrying individuals (secretors). Perhaps the $H$. pylori stimulates, or even catalyses, the synthesis of one or more novel structures, at the same time more effectively blocking the epitope recognised by LICRLonM8. It is noteworthy that several of the same carbohydrate structures also occur on the $H$. pylori organisms themselves, as well as the enzymes to synthesise them (Wang et al, 1999; Rasko et al, 2000; Wang et al, 2000; Nilsson et al, 2006, 2008), and there is clearly a subtle host-bacteria interaction.

In the context of cancer, despite extensive literature mentioning aberrant glycosylation of MUC1, very little has been done experimentally with deglycosylation of MUC1 in situ. An exception to this is the case of MUSE-11, where deglycosylation was shown to reveal cell surface as well as intracellular MUC1 in gastric cancer (Bara et al, 1993; Hinoda et al, 1998). Two other mAbs to the TR domain of MUC1 (HMFG1 and SM3) have been shown to differ in their intracellular and apical detection of MUC1 in gastric cancer, which has been attributed to the extent of glycosylation of the epitope detected by these reagents (Reis et al, 1998), though deglycosylation was not done.

More broadly this paper highlights the issue of epitope specificity of monoclonal antibodies directed against diseaseassociated markers, specifically when they are glycoproteins. This is of particular importance to cancer studies, as changes in glycosylation are common in cancer. The simple strategy of periodate treatment helps to interpret such changes in epitope expression. In the case of the MUC1 tandem repeat domain there are clearly subtleties in epitope specificity of all the reagents even when they apparently recognise the core peptide. Indeed it has previously been shown that some MUC1 mAbs have increased binding to peptides with simple substitution on the T of DTR with galactose or the disaccharide Gal GalNac (Karsten et al, 1998). There are several other important examples in the literature of cancer-related antigens used in diagnostic tests and therapeutic approaches that are located on glycoproteins (e.g., CEA, CA125/ MUC16) for which the same issues may apply, namely, changes in crypticity of epitopes may be confused with changes in gene expression or level of secretion. The results presented here provide a caveat for such interpretations and suggest that the simple strategy of periodate treatment should be used more frequently to follow up on apparent changes in expression.

\section{ACKNOWLEDGEMENTS}

We thank Dr Adil Elamin for assistance with collection of the samples used in this study and Drs Sandy Gendler, Mike
McGuckin and Kohzoh Imai for the kind donation of their antibody reagents; LICRLonM8 was originally produced by the Lister Institute for Cancer Research. This work was supported by the UCL Experimental Cancer Medicine Centre, funded by CRUK and the National Institute for Health Research (NIHR).

\section{REFERENCES}

Bara J, Imberty A, Perez S, Imai K, Yachi A, Oriol R (1993) A fucose residue can mask the MUC-1 epitopes in normal and cancerous gastric mucosae. Int J Cancer 54(4): 607-613.

Beatson RE, Taylor-Papadimitriou J, Burchell JM (2010) MUC1 immunotherapy. Immunotherapy 2(3): 305-327.

Croce MV, Isla-Larrain MT, Rua CE, Rabassa ME, Gendler SJ, Segal-Eiras A (2003) Patterns of MUC1 tissue expression defined by an anti-MUC1 cytoplasmic tail monoclonal antibody in breast cancer. J Histochem Cytochem 51(6): 781-788.

Dixon MF, Genta RM, Yardley JH, Correa P (1996) Classification and grading of gastritis. The updated Sydney System. International Workshop on the Histopathology of Gastritis, Houston 1994. Am J Surg Pathol 20(10): 1161-1181.

Hinoda Y, Arimura Y, Itoh F, Adachi M, Tsujisaki M, Imai K, Yachi A (1993) Primary structure of the variable regions of a monoclonal antibody MUSE11 recognizing the tandem repeat domain of a mucin core protein, MUC1. J Clin Lab Anal 7(2): 100-104.

Hinoda Y, Nakagawa N, Ohe Y, Kakiuchi H, Tsujisaki M, Imai K, Yachi A (1990) Recognition of the polypeptide core of mucin by monoclonal antibody MUSE11 against an adenocarcinoma-associated antigen Jpn J Cancer Res 81(12): 1206-1209.

Hinoda Y, Takahashi T, Hayashi T, Suwa T, Makiguchi Y, Itoh F, Adachi M, Imai K (1998) Enhancement of reactivity of anti-MUC1 core protein antibody and killing activity of anti-MUC1 cytotoxic T cells by deglycosylation of target tissues or cells. J Gastroenterol 33(2): 164-171.

Karsten U, Diotel C, Klich G, Paulsen H, Goletz S, Muller S, Hanisch FG (1998) Enhanced binding of antibodies to the DTR motif of MUC1 tandem repeat peptide is mediated by site-specific glycosylation. Cancer Res 58(12): 2541-2549.

Kufe DW (2009) Mucins in cancer: function, prognosis and therapy. Nat Rev Cancer 9(12): 874-885.

Linden S, Boren T, Dubois A, Carlstedt I (2004a) Rhesus monkey gastric mucins: oligomeric structure, glycoforms and Helicobacter pylori binding. Biochem J 379(Pt 3): 765-775.

Linden S, Mahdavi J, Hedenbro J, Boren T, Carlstedt I (2004b) Effects of pH on Helicobacter pylori binding to human gastric mucins: identification of binding to non-MUC5AC mucins. Biochem J 384(Pt 2): 263-270.

Linden SK, Sheng YH, Every AL, Miles KM, Skoog EC, Florin TH, Sutton P, McGuckin MA (2009) MUC1 limits Helicobacter pylori infection both by steric hindrance and by acting as a releasable decoy. PLoS Pathog 5(10): e1000617.

McGuckin MA, Every AL, Skene CD, Linden SK, Chionh YT, Swierczak A, McAuley J, Harbour S, Kaparakis M, Ferrero R, Sutton P (2007) Muc1 mucin limits both Helicobacter pylori colonization of the murine gastric mucosa and associated gastritis. Gastroenterology 133(4): 1210-1218.

McIlhinney RA, Patel S, Gore ME (1985) Monoclonal antibodies recognizing epitopes carried on both glycolipids and glycoproteins of the human milk fat globule membrane. Biochem J 227(1): 155-162.

Mukhopadhyay P, Chakraborty S, Ponnusamy MP, Lakshmanan I, Jain M, Batra SK (2011) Mucins in the pathogenesis of breast cancer: implications in diagnosis, prognosis and therapy. Biochim Biophys Acta 1815(2): 224-240.

Nilsson C, Skoglund A, Moran AP, Annuk H, Engstrand L, Normark S (2006) An enzymatic ruler modulates Lewis antigen glycosylation of Helicobacter pylori LPS during persistent infection. Proc Natl Acad Sci USA 103(8): 2863-2868.

Nilsson C, Skoglund A, Moran AP, Annuk H, Engstrand L, Normark S (2008) Lipopolysaccharide diversity evolving in Helicobacter pylori communities through genetic modifications in fucosyltransferases. PLoS One 3(11): e3811.

Ota H, Nakayama J, Momose M, Hayama M, Akamatsu T, Katsuyama T, Graham DY, Genta RM (1998) Helicobacter pylori infection produces reversible glycosylation changes to gastric mucins. Virchows Arch 433(5): 419-426. 
Price MR, Edwards S, Owainati A, Bullock JE, Ferry B, Robins RA, Baldwin RW (1985) Multiple epitopes on a human breast-carcinomaassociated antigen. Int J Cancer 36(5): 567-574.

Price MR, Hudecz F, O'Sullivan C, Baldwin RW, Edwards PM, Tendler SJ (1990) Immunological and structural features of the protein core of human polymorphic epithelial mucin. Mol Immunol 27(8): 795-802.

Rasko DA, Wang G, Monteiro MA, Palcic MM, Taylor DE (2000) Synthesis of mono- and di-fucosylated type I Lewis blood group antigens by Helicobacter pylori. Eur J Biochem 267(19): 6059-6066.

Reis CA, David L, Seixas M, Burchell J, Sobrinho-Simoes M (1998) Expression of fully and under-glycosylated forms of MUC1 mucin in gastric carcinoma. Int J Cancer 79(4): 402-410.

Rong Y, Qin X, Jin D, Lou W, Wu L, Wang D, Wu W, Ni X, Mao Z, Kuang T, Zang YQ, Qin X (2012) A phase I pilot trial of MUC1-peptide-pulsed dendritic cells in the treatment of advanced pancreatic cancer. Clin Exp Med 12(3): 173-180.

Schroeder JA, Thompson MC, Gardner MM, Gendler SJ (2001) Transgenic MUC1 interacts with epidermal growth factor receptor and correlates with mitogen-activated protein kinase activation in the mouse mammary gland. J Biol Chem 276(16): 13057-13064.

Taylor-Papadimitriou J, Burchell J, Miles DW, Dalziel M (1999) MUC1 and cancer. Biochim Biophys Acta 1455(2-3): 301-313.
Vinall LE, King M, Novelli M, Green CA, Daniels G, Hilkens J, Sarner M, Swallow DM (2002) Altered expression and allelic association of the hypervariable membrane mucin MUC1 in Helicobacter pylori gastritis. Gastroenterology 123(1): 41-49.

Wang G, Boulton PG, Chan NW, Palcic MM, Taylor DE (1999) Novel Helicobacter pylori alpha1,2-fucosyltransferase, a key enzyme in the synthesis of Lewis antigens. Microbiology 145(Pt 11): 3245-3253.

Wang G, Ge Z, Rasko DA, Taylor DE (2000) Lewis antigens in Helicobacter pylori: biosynthesis and phase variation. Mol Microbiol 36(6): 1187-1196.

Xing PX, Prenzoska J, McKenzie IF (1992) Epitope mapping of anti-breast and anti-ovarian mucin monoclonal antibodies. Mol Immunol 29(5): 641-650.

Xing PX, Tjandra JJ, Stacker SA, Teh JG, Thompson CH, McLaughlin PJ, McKenzie IF (1989) Monoclonal antibodies reactive with mucin expressed in breast cancer. Immunol Cell Biol 67(Pt 3): 183-195.

This work is published under the standard license to publish agreement. After 12 months the work will become freely available and the license terms will switch to a Creative Commons AttributionNonCommercial-Share Alike 3.0 Unported License.

Supplementary Information accompanies the paper on British Journal of Cancer website (http://www.nature.com/bjc) 